\title{
A Cross-Sectional Study of Oral Lichen Planus Associated With Thyroid Diseases in East China
}

\begin{abstract}
Yunju Tang ${ }^{1,2 t}$, Linjun Shi ${ }^{1,2 t}$, Boren Jiang ${ }^{3}$, Zengtong Zhou ${ }^{1,2}$ and Xuemin Shen ${ }^{1,2 *}$
${ }^{1}$ Department of Oral Mucosal Diseases, Shanghai Ninth People's Hospital, College of Stomatology, Shanghai Jiao Tong University School of Medicine, Shanghai, China, ${ }^{2}$ National Clinical Research Center for Oral Diseases, Shanghai Key Laboratory of Stomatology \& Shanghai Research Institute of Stomatology, Shanghai, China, ${ }^{3}$ Department of Endocrinology and Metabolism, Shanghai Ninth People's Hospital, Shanghai Jiao Tong University School of Medicine, Shanghai, China
\end{abstract}

Objective: To investigate the prevalence of thyroid diseases in patients with oral lichen planus (OLP) and to explore the correlation between the two diseases.

Methods: A cross-sectional study was conducted to investigate the history of thyroid disease in 585 patients with oral lichen planus diagnosed in the Department of Oral Mucosal Diseases of the Ninth People's Hospital, Shanghai Jiaotong University, School of Medicine from June 2017 to April 2018 and in 10,441 normal people in

OPEN ACCESS

Edited by:

Noriyuki Koibuchi, Gunma University, Japan

Reviewed by:

Pia Lopez Lopez Jornet, University of Murcia, Spain

Fabio Camacho-Alonso,

University of Murcia, Spain

*Correspondence:

Xuemin Shen

kiyoshen@163.com

tThese authors have contributed equally to this work

Specialty section:

This article was submitted to

Thyroid Endocrinology,

a section of the journal

Frontiers in Endocrinology

Received: 25 September 2019 Accepted: 19 December 2019

Published: 24 January 2020

Citation:

Tang Y, Shi L, Jiang B, Zhou Z and Shen X (2020) A Cross-Sectional

Study of Oral Lichen Planus Associated With Thyroid Diseases in East China. Front. Endocrinol. 10:928

doi: 10.3389/fendo.2019.00928 an epidemiological survey conducted by endocrinology department of Ninth People's Hospitalin eastern China from 2014 to 2015. Personal medical history of thyroid disease was obtained through questionnaire and thyroid function was also tested.

Results: Of the 585 patients with OLP, 190 (32.48\%) had thyroid disease (excluding coexistence of multiple thyroid diseases), 62 (32.6\%) had thyroid nodules, and 71 (37.4\%) had Hashimoto's thyroiditis. Hyperthyroidism was diagnosed in six patients (3.2\%), hypothyroidism in seven patients (3.7\%), and thyroid cancer in 11 patients (5.8\%). The prevalence of Hashimoto's thyroiditis was significantly higher in patients with oral lichen planus than in the general population. The probability of thyroid disease was significantly higher in women with OLP than in men with OLP $(P<0.001)$.

Conclusion: OLP is associated with a high probability of developing thyroid disease, especially Hashimoto's thyroiditis. In the management of OLP patients, especially in female patients, thyroid disease must be screened.

Keywords: oral lichen planus, thyroid disease, Hashimoto's thyroiditis, immunology, cross-sectional study

\section{INTRODUCTION}

Oral Lichen Planus (OLP) is a chronic inflammatory oral mucosal disease, which is common in middle-aged women (1). The clinical features of OLP is multifocal, usually bilateral affecting buccal mucosa, tongue, lips, gingiva, and white reticular patches with or without erosions and ulcerations. The histopathological features of OLP is Hyperkeratosis with basal cell degeneration, necrosis of basal, and parabasal keratinocytes, and a band-like predominantly lymphocytic infiltrate adjacent to basal cells (2). However, the etiology and pathogenesis of OLP are still enigmatic. Several studies indicated that OLP is closely related to some systemic diseases, such as diabetes mellitus (DM), hepatitis $C$ and thyroid diseases $(3,4)$. Among these diseases, some scholars have shown that the 
immune system may play a critical role in the appearance of OLP in patients with type I DM (5). Thyroid diseases (TD) such as hyperthyroidism, thyroid cancer, and Hashimoto's thyroiditis (HT) have a diverse prevalence ranging from 0.5 to $1 \%$ in Europe, Japan, and the USA (6). However, in China, due to the huge population base, over 200 million people suffer from TD, where the prevalence of hypothyroidism and hyperthyroidism has been estimated to be 6.5 and $1.3 \%$, respectively (7). In 2016, a study was conducted by Chinese Society of Endocrinology, revealing that more than $40 \%$ of the population suffered from TD (8). In the study of the relationship between OLP and TD, some scholars found that erosive OLP has been associated with anti-TPO autoantibodies (TPOAb) in thyroid patients, it may be useful to determine TPOAb levels of such patients to diagnose a possible undetected thyroid disorders and followup for malignancy (9). HT is a kind of TD with incidence from 0.4 to $2 \%$ in the general population (10). HT often causes thyroid dysfunction, especially hypothyroidism, and some HT patients require surgical treatment, which increases the burden of disease treatment. So, more attention should be paid to HT (11). Several studies in other populations have investigated the possible relationship between thyroid diseases and OLP (12-14). Only one literature from China reported the possible relationship between OLP and TD in northern Chinese population, and this study included $<200$ patients with oral lichen planus (15). However, the epidemiological characteristics of OLP with HT have never been discussed. Therefore, a larger sample size study was needed to enrich the knowledge of association between OLP and TD, especially HT.

\section{MATERIALS AND METHODS}

The present study was approved by the ethics committee of Shanghai Ninth People's Hospital (2016-201-T145), and written informed consent was obtained from all participants. A crosssectional study was conducted to investigate 585 patients with oral lichen planus from June 2017 to April 2018. The personal medical history of thyroid disease was obtained by questionnaire, and thyroid function including thyroid peroxidase antibody (TPOAb), thyroglobulin (TGAb), thyroid-stimulating hormone (TSH), free triiodothyronine (FT3), free thyroxine (FT4) was also tested. Thyroid disease information of the 10,441 normal people as a control group came from an epidemiological survey conducted by endocrinology department of Shanghai Ninth People's Hospital in east China from 2014 to 2015. The gender, age, TPOAb, TGAb, TSH, FT3, FT4, and B-ultrasound results of these people are all from the epidemiological investigation (16).

\section{Inclusion Criteria}

OLP patients who were diagnosed clinically and histologically according to the diagnostic criteria of AAOMP (17). Briefly, for the diagnosis of OLP, the clinical criteria included the presence of bilateral, more or less symmetric lesions; the lesions could be lace-like network of gray-white lines, plaque, atrophic, bullous, or erosive type. The histological criteria included the presence of a distinct band-like zone of cellular infiltration confined to the superficial part of the connective tissue, a sign of liquefaction degeneration in the basal cell layer and the absence of epithelial dysplasia. According to the guideline of diagnosis and treatment of thyroid diseases in China (18), the criteria for Hashimoto's thyroiditis (HT) included the positive detection of thyroid peroxidase antibody (TPOAb) and thyroglobulin (TGAb), with or without local and systemic manifestations including dysphonia, dyspnea, and constipation (19). For hypothyroidism, it included the higher value of thyroid-stimulating hormone (TSH), the lower value of total thyroxine (TT4), and free thyroxine (FT4). For hyperthyroidism, it included the lower value of TSH, the higher value of TT4, FT4, total triiodothyronine (TT3), and free triiodothyronine (FT3), with or without clinical symptoms and signs of clinical hypermetabolism, such as increased heart rate. For thyroid nodules, it included one or more lump in the thyroid gland detected by B ultrasound.

\section{Exclusion Criteria}

Patients under 18 years old or who were pregnant; patients with a history of action capability restricting or life-threatening systemic diseases like uremia, or other autoimmune diseases which seriously affects the quality of life, such as psoriasis, vitiligo, Behcet disease, or bullous diseases.

\section{Data Collection}

The demographic and clinical information of the participants was recorded including age, gender, the score of clinical manifestation, medication history, and general condition. No more thyroid examination including B-ultrasound was performed for participants with diagnosed thyroid diseases. On the contrary, if the participants had not been diagnosed with any thyroid disease in the past, these participants were instructed to undergo a thyroid examination including thyroid function (FT3, FT4, and TSH), thyroid related antibodies (TPOAb and TGAb). These indicators were detected by chemiluminescence immunoassay (CLIA). And, these participants also received a B-ultrasound of the thyroid gland (20). Results of the thyroid examination were determined by two independent endocrinologists of Shanghai Ninth People's Hospital to confirm the diagnosis of HT, hypothyroidism, thyroid nodule, and hyperthyroidism accordingly.

\section{Statistical Analysis}

The data were processed by SPSS 23.0 software package. We calculated the odds ratio (OR) with $95 \%$ confidence intervals (CI) for associations between OLP and control group in different types of thyroid diseases. Comparison of parameters of age and OLP score between the OLP and control group was performed by $T$-test. Comparison of parameters of Gender, Smoking history and Clinical type between the OLP and control group was performed by $\chi^{2}$ test. The difference was statistically significant with $P<0.05$.

\section{RESULTS}

The proportion of male and smoking history in OLP patients with TD was significantly lower than that in patients without 
TD (Table 1). Rate of different thyroid diseases (excluding coexistence of multiple thyroid diseases) in OLP patients with TD (Figure 1, Table 2). The proportion of female OLP patients was higher than that in control group (Table 3). HT ranked first in the OLP-associated TD, followed by thyroid nodules. The prevalence of HT and Multiple in OLP patients was significantly higher than that in control group. The prevalence of Thyroid nodule and Hypothyroidism in OLP patients was significantly lower than that in control group (Table 4). Among female, the prevalence of HT in OLP patients was significantly higher than that in control group, but there was no difference among male (Table 5). Considering HT was the most common type of TD in OLP patients, so we specially analyzed the situation of OLP with HT. Similar to the results in Table 1, the two groups of

TABLE 1 | Difference of demographics information and clinical information between OLP with TD and OLP without TD.

\begin{tabular}{lccc}
\hline & OLP with TD & OLP without TD & $P$-value \\
\hline Number & 190 & 395 & \\
GENDER & & & \\
Male (\%) & $23(12.1)$ & $121(30.6)$ & $P<0.001$ \\
Female (\%) & $167(87.9)$ & $274(69.4)$ & \\
Age & $52.85 \pm 13.15$ & $52.72 \pm 13.71$ & $P>0.05$ \\
Smoking history (\%) & $26(13.7)$ & $126(31.9)$ & $P<0.001$ \\
CLINICAL TYPE & & & \\
Erosive (\%) & $26(13.7)$ & $44(11.1)$ & $P>0.05$ \\
Non-erosive (\%) & $164(86.3)$ & $351(88.9)$ & \\
OLP score & $4.70 \pm 2.37$ & $4.56 \pm 2.50$ & $P>0.05$
\end{tabular}

patients also had obvious differences in gender and smoking history (Table 6).

\section{DISCUSSION}

Although the pathogenesis of OLP is still unclear, recently, more and more studies have shown that OLP is related to systemic diseases such as autoimmune diseases, endocrine diseases, and metabolic diseases $(1,21)$. Researchers in China and other countries have paid much attention to thyroid diseases among all kind of systemic diseases, but most of them have few cases and fewer thyroid diseases (22). However, study from China with large sample still lacked evidence of the relationship between OLP
TABLE 2 | Rate of different thyroid diseases (excluding coexistence of multiple thyroid diseases) in OLP patients with TD.

\begin{tabular}{lcc}
\hline $\begin{array}{l}\text { Different thyroid diseases } \\
\text { among OLP patients }\end{array}$ & Number & $\begin{array}{c}\text { Rate of different thyroid } \\
\text { diseases in OLP patients } \\
\text { with TD, \% }\end{array}$ \\
\hline Thyroid nodule & $62^{\mathrm{a}}$ & 32.6 \\
Hashimoto's thyroiditis & 71 & 37.4 \\
Hyperthyroidism & 6 & 3.2 \\
Hypothyroidism & 7 & 3.7 \\
Thyroid cancer & 11 & 5.8 \\
Multiple & 33 & 17.4 \\
\hline Total & 190 & 100 \\
\hline
\end{tabular}

a Sixty-two patients with thyroid nodules in OLP patients were from 212 patients who received a $B$-ultrasound of the thyroid gland.

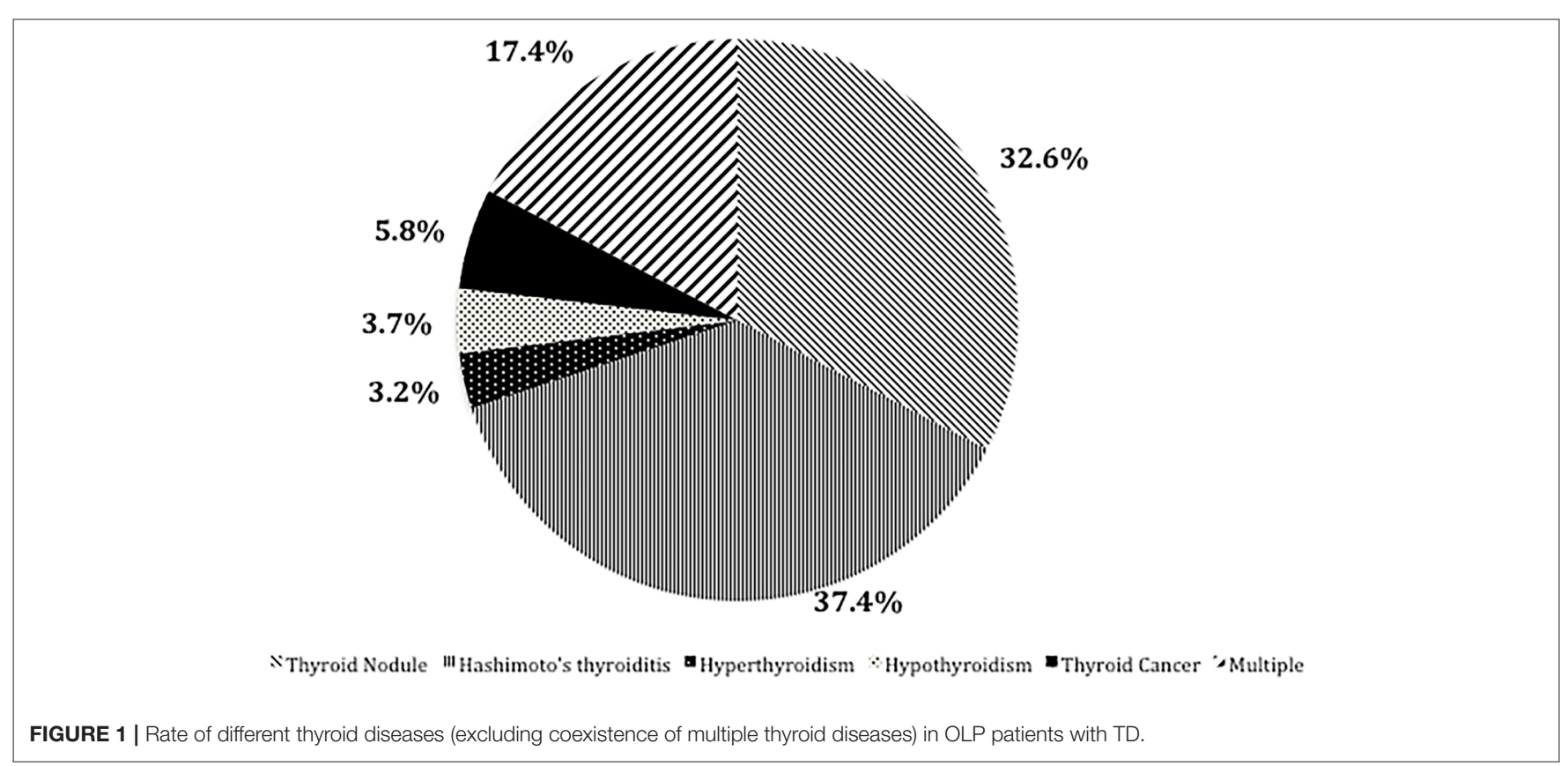


and thyroid diseases, hence the present study was designed to enrich the knowledge. The majority of OLP patients we collected were middle-aged and elderly female, which was consistent with the characteristics of OLP in the past study (23). Among the systemic diseases associated with OLP, more attention to TD was paid. The incidence of TD is higher than the results of other researchers but not significantly higher than that of the general population $(p>0.05)$. In the present study, female OLP patients older than 40 years old were more likely to suffer from TD probably because both OLP and thyroid diseases were prevalent among middle-aged and elderly females.

This study expanded the sample size and focused on the classification of thyroid diseases associated with OLP patients. Among OLP with TD, the present study found that Hashimoto thyroiditis and Thyroid nodule were prevalent.

In the present manuscript, although HT is the most common thyroid disease among OLP patients, OLP with HT has no particularity in OLP with TD in terms of demographic information and clinical manifestations. Based on its own research and the results of literature review, it screened out the more possible mechanisms from the respective pathogenesis of the two diseases and discussed them. There are roughly the following theories: (1) HT is mostly originated from OLP. Some scholars speculated that the changes in hormone levels and thyroid autoantibodies caused by HT may lead to changes in oral local keratinocytes, thus triggering OLP (24). (2) The peripheral blood and oral lesions of OLP patients showed $\mathrm{T}$ lymphocyte distribution different from that of healthy people, with abnormal increase of cytokines TNF- $\alpha$ and IFN- $\gamma$ (25, 26). The peripheral blood of HT patients also showed similar phenomenon $(27,28)$, so this immunological change may be

TABLE 3 | Demographics information between OLP patients and control group.

\begin{tabular}{lccc}
\hline & OLP & control group & $P$-value \\
\hline Number & 585 & 10,441 & \\
GENDER & & & \\
Male (\%) & $144(24.6)$ & $4,310(41.3)$ & $P<0.05$ \\
Female (\%) & $441(75.4)$ & $6,131(58.7)$ & \\
Age & $52.80 \pm 13.52$ & $53.50 \pm 13.05$ & $P>0.05$
\end{tabular}

the common pathogenesis of both, and OLP and HT may also be two clinical manifestations of a certain syndrome. (3) In terms of genetic factors, there were also relevant researches on the susceptibility genes of OLP and HT. The coexistence of the two susceptibility genes may also be the cause of the two diseases, but the relationship between the two susceptibility genes has not yet been determined (29). Since the pathogenesis of HT and OLP was similar, both of which were T cell mediated local immune responses, so the relationship between the two needed more attention, and cellular immunity might be a better research direction. Since it is impossible to distinguish whether the results are caused by OLP alone or HT or by a combination of two diseases, although the pathogenesis of OLP and HT has

TABLE 5 | The prevalence of HT in OLP patients and the normal people was compared between male and female, respectively.

\begin{tabular}{lcccc}
\hline Gender & Group & $\begin{array}{c}\text { Number and rate of HT in } \\
\text { OLP patients }\end{array}$ & $\begin{array}{c}\text { Number and rate } \\
\text { of HT in normal } \\
\text { people }\end{array}$ & P-value \\
\hline Male & HT & $8(5.6 \%)$ & $134(3.1 \%)$ & $P>0.05$ \\
& Total & 144 & 4,310 & \\
Female & HT & $63(14.3 \%)$ & $504(8.2 \%)$ & $P<0.05$ \\
& Total & 441 & 6,131 & \\
\hline
\end{tabular}

TABLE 6 | Demographics information and clinical information from the OLP with HT and OLP without TD.

\begin{tabular}{lccc}
\hline & OLP with HT & OLP without TD & $P$-value \\
\hline Number & 97 & 395 & \\
GENDER & $10(10.3)$ & $121(30.6)$ & $P<0.001$ \\
Male (\%) & $87(89.7)$ & $274(69.4)$ & \\
Female (\%) & $50.77 \pm 13.44$ & $52.72 \pm 13.71$ & $P>0.05$ \\
Age & $11(11.3)$ & $126(31.9)$ & $P<0.001$ \\
Smoking history (\%) & $13(13.4)$ & $44(11.1)$ & $P>0.05$ \\
CLINICAL TYPE & $84(86.6)$ & $351(88.9)$ & \\
Erosive (\%) & $4.71 \pm 2.36$ & $4.56 \pm 2.50$ & $P>0.05$ \\
Non-erosive (\%) & & & \\
OLP score & & &
\end{tabular}

TABLE 4 | Number and rate of different thyroid diseases (excluding coexistence of multiple thyroid diseases) in 585 OLP patients and control group.

\begin{tabular}{|c|c|c|c|c|c|c|}
\hline $\begin{array}{l}\text { Different thyroid } \\
\text { diseases among OLP } \\
\text { patients }\end{array}$ & Number & $\begin{array}{l}\text { Rate of different } \\
\text { thyroid diseases in } \\
\text { OLP patients, \% }\end{array}$ & Number & $\begin{array}{c}\text { Population rate } \\
\text { of control group, } \\
\%\end{array}$ & $P$-value & $\begin{array}{c}\text { OR }[95 \% \text { confidence } \\
\text { intervals }(\mathrm{CI})]\end{array}$ \\
\hline Thyroid nodule & $62^{a}$ & 10.6 & 5,183 & 49.6 & $P<0.05$ & $0.120(0.092,0.157)$ \\
\hline Hashimoto's thyroiditis & 71 & 12.1 & 638 & 6.1 & $P<0.05$ & $2.122(1.635,2.755)$ \\
\hline Hyperthyroidism & 6 & 1.0 & 48 & 0.5 & $P>0.05$ & $2.244(0.956,5.264)$ \\
\hline Hypothyroidism & 7 & 1.2 & 430 & 4.1 & $P<0.05$ & $0.282(0.133,0.598)$ \\
\hline Thyroid cancer & 11 & 1.9 & 117 & 1.1 & $P>0.05$ & $1.691(0.906,3.155)$ \\
\hline Multiple & 33 & 5.6 & 193 & 1.8 & $P<0.05$ & $3.174(2.173,4.637)$ \\
\hline Other thyroid diseases & NA & NA & 22 & 0.2 & NA & NA \\
\hline
\end{tabular}

a Sixty-two patients with thyroid nodules in OLP patients were from 212 patients who received a B-ultrasound of the thyroid gland. 
many similarities, it is still necessary to study the pathogenesis of OLP and HT. And more attention should be paid to the primary and secondary diseases, which is the main obstacle for our future research.

The thyroid disease information of some patients with OLP came from the medical history, and the information of the other patients came from the examinations that were done at that time. Although the prevalence of thyroid nodules was significantly different between the two groups, 62 patients with thyroid nodules in OLP patients were from 212 patients who received B-ultrasound of the thyroid gland, the prevalence of thyroid nodules in OLP patients was not representative. Thyroid nodules ranked second in the prevalence of thyroid disease associated with OLP patients. Thyroid nodules are common, being detected in up to $65 \%$ of the general population. Most thyroid nodules are benign, clinically insignificant, and safely managed with a surveillance program. However, this nodule that harbor a clinically significant cancer $(\approx 10 \%)(30)$. Thyroid cancer is one of the cancers with lower mortality rate, but it ranks fourth in the incidence of women (31). Therefore, it is necessary to discover thyroid cancer in advance. Among patients with OLP, 11 patients developed thyroid cancer after OLP. The thyroid cancer in these patients was caused by malignant transformation of thyroiditis or thyroid nodules, often accompanied by other thyroid diseases. This phenomenon deserves our close attention. Although the mortality rate of thyroid cancer is not high, the incidence rate of thyroid cancer is increasing, including malignant transformation of various thyroid diseases and primary thyroid cancer, so the economic burden of the disease is also increasing (32). It is still too early to use OLP as an early warning indicator for thyroid cancer. However, examination of serum thyroid function and thyroid B ultrasound examination in OLP patients is necessary for the diagnosis of thyroid disease in order to find thyroid cancer at an early stage, especially for the elderly and women.

The limitation in this study is that the data of the control group are historical data and there is no comparison between the data at the same time.

\section{REFERENCES}

1. Eisen D, Carrozzo M, Bagan Sebastian JV, Thongprasom K. Number V Oral lichen planus: clinical features and management. Oral Dis. (2005) 11:33849. doi: 10.1111/j.1601-0825.2005.01142.x

2. Roopashree MR, Gondhalekar RV, Shashikanth MC, George J, Thippeswamy $\mathrm{SH}$, Shukla A. Pathogenesis of oral lichen planus - a review. J Oral Pathol Med. (2010) 39:729-34. doi: 10.1111/j.1600-0714.2010.00946.x

3. Zhao W, Yang Y, Wu F, Zhou H. The reciprocal association between diabetes mellitus and erosive oral lichen planus. Oral Dis. (2019) 25:12356. doi: 10.1111/odi.13050

4. Compilato D, Paderni C, Di Fede O, Gulotta G, Campisi G. Association of oral lichen planus with thyroid disease in a Finnish population: a retrospective case-control study. Oral Surg Oral Med Oral Pathol Oral Radiol Endod. (2011) 111:12-3. doi: 10.1016/j.tripleo.2010.09.074

5. Petrou-Amerikanou C, Markopoulos AK, Belazi M, Karamitsos D, Papanayotou P. Prevalence of oral lichen planus in diabetes mellitus according to the type ofdiabetes. Oral Dis. (1998) 4:37-40. doi: 10.1111/j.1601-0825.1998.tb00253.x

\section{CONCLUSION}

To sum up, the present study showed that OLP was closely related to HT through a larger sample size. The possible mechanism behind this correlation is still enigmatic and deserves further research. Middle-aged women with OLP needed to undergo thyroid blood tests and B ultrasound to screen for thyroid diseases.

\section{DATA AVAILABILITY STATEMENT}

All datasets generated for this study are included in the article/supplementary material.

\section{ETHICS STATEMENT}

The studies involving human participants were reviewed and approved by the ethics committee of Shanghai Ninth People's Hospital (2016-201-T145). The patients/participants provided their written informed consent to participate in this study.

\section{AUTHOR CONTRIBUTIONS}

YT and XS developed the project and implementation scheme. YT, BJ, and XS recruited and collected clinical cases. YT performed the statistical analysis of relevant data. YT and LS participated in the writing and modification of the article. XS and $\mathrm{ZZ}$ critically revised the manuscript.

\section{FUNDING}

This study was supported by the National Key R\&D Program of China (2017YFC0840100, 2017YFC0840110); Clinical Research Program of 9th People's Hospital, Shanghai Jiao Tong University School of Medicine (JYLJ008); Clinical Research Project of MultiDisciplinary Team, Shanghai Ninth People's Hospital, Shanghai JiaoTong University School of Medicine (201701006); Oral Mucosal Disease of Traditional Chinese Medicine.

6. Vanderpump MP. The epidemiology of thyroid disease. Br Med Bull. (2011) 99:39-51. doi: 10.1093/bmb/ldr030

7. Yang K. The number of thyroid patients in China is over 200 million (in chinese). Shanghai Med Pharm. (2013) 34:44.

8. Shan Z, Chen L, Lian X, Liu C, Shi B, Shi L., et al. The iodine status and prevalence of thyroid disorders after introduction of mandatory universal salt iodization for 16 years in China A cross-sectional study in 10 cities. Thyroid. (2016) 26:1125-30. doi: 10.1089/thy.2015.0613

9. Alikhani M, Ghalaiani P, Askariyan E, Khunsaraki ZA, Tavangar A, Naderi A. Association between the clinical severity of oral lichen planus and anti-TPOlevel in thyroid patients. Braz Oral Res. (2017) 31:e10. doi: 10.1590/1807-3107bor-2017.vol31.0010

10. Ahmed R, Al-Shaikh S, Akhtar M. Hashimoto thyroiditis: a century later. Adv AnatPathol. (2012) 19:181-6. doi: 10.1097/PAP.0b013e31825 34868

11. Zivaljevic VR, Bukvic Bacotic BR, Sipetic SB, Stanisavljevic DM, Maksimovic JM, Diklic AD, et al. Quality of life improvement in patients with Hashimoto thyroiditis and other goiters after surgery: a prospective cohort study. Int J Surg. (2015) 21:150-5. doi: 10.1016/j.ijsu.2015.08.001 
12. Fabrizio G, Roberta G, Flavia DB, Cannavò SP, Benvenga S. Thyroid autoimmunity and lichen. Front Endocrinol. (2017) 27:146. doi: 10.3389/fendo.2017.00146

13. Garcia-Pola MJ, Llorente-Pendás S, Seoane-Romero JM, Berasaluce MJ, García-Martín JM. Thyroid disease and oral lichen planus as comorbidity: a prospective case-control study. Dermatology. (2016) 232:214-9. doi: 10.1159/000442438

14. Lavaee F, Majd M. Evaluation of the association between oral lichen planus and hypothyroidism: a retrospective comparative study (Shiraz). J Dentist. (2016) 17:38-42.

15. Zhou T, Li D, Chen Q, Hua H, Li C. Correlation between oral lichen planus and thyroid disease in china: a case-control study. Front Endocrinol. (2018) 18:330. doi: 10.3389/fendo.2018.00330

16. Chen Y, Wang N, Chen Y, Li Q, Han B, Chen C, et al. The association of non-alcoholic fatty liver disease with thyroid peroxidase and thyroglobulin antibody: A new insight from SPECT-China study. Autoimmunity. (2018) 51:238-44. doi: 10.1080/08916934.2018.1488968

17. Cheng YS, Gould A, Kurago Z, Fantasia J, Muller S. Diagnosis of oral lichen planus: a position paper of the American Academy of Oral and Maxillofacial Pathology. Oral Med Oral Pathol Oral Radiol. (2016) 122:33254. doi: 10.1016/j.0ooo.2016.05.004

18. Chinese Society of Endocrinology. A guide to the diagnosis and treatment of thyroid diseases in China-laboratory and auxiliary examinations for thyroid diseases (in chinese). Chin J Intern Med. (2007) 46:697-702. doi: 10.3760/j.issn:0578-1426.2007.08.038

19. Caturegli P, De RA, Rose NR. Hashimoto thyroiditis: clinical and diagnostic criteria. Autoimmun Rev. (2014) 13:391-7. doi: 10.1016/j.autrev.2014.01.007

20. Baloch Z, Carayon P, Conte-Devolx B, Demers LM, Feldt-Rasmussen U, Henry JF, et al. Laboratory medicine practice guidelines. Laboratory support for the diagnosis and monitoring of thyroid disease. Thyroid. (2003) 13:3. doi: 10.1089/105072503321086962

21. Srinivas K, Aravinda K, Ratnakar P, Nigam N, Gupta S. Oral lichen planus-Review on etiopathogenesis. Natl J Maxil Surg. (2011) 2:156. doi: 10.4103/0975-5950.85847

22. Lo Muzio L, Santarelli A, Campisi G, Lacaita M, Favia G. Possible link between Hashimoto's thyroiditis and oral lichen planus:a novel association found. Clin Oral Investig. (2013) 17:333-6. doi: 10.1007/s00784-0120767-4

23. Alrashdan MS, Cirillo N, McCullough M. Oral lichen planus: a literature review and update. Arch Dermatol Res. (2016) 308:539-51. doi: 10.1007/s00403-016-1667-2
24. Slominski A, Wortsman J, Kohn L, Ain KB, Venkataraman GM, Pisarchik A, et al. Expression of hypothalamic-pituitary-thyroid axis related genes in the human skin. J Invest Dermatol. (2002) 119:1449-55. doi: 10.1046/j.1523-1747.2002.19617.x

25. Javvadi LR, Parachuru VP, Milne TJ, Seymour GJ, Rich AM. Regulatory Tcells and IL17A(+) cells infiltrate oral lichen planus lesions. Pathology. (2016) 48:564-73. doi: 10.1016/j.pathol.2016.06.002

26. Piccinni MP, Lombardelli L, Logiodice F, Tesi D, Kullolli O, Biagiotti R, et al. Potential pathogeneticrole of Th17, Th0, and Th2 cells in erosive and reticular oral lichen planus. Oral Dis. (2014) 20:212-8. doi: 10.1111/odi.12094

27. Ju SH. Relationship between Th1/Th2 cell imbalance and autoantibody production in hashimoto's thyroiditis patients. Contemp Med. (2007) 12:4951. doi: 10.1016/j.cellbi.2008.01.216

28. Liu Y, Tang X, Tian J, Zhu C, Peng H, Rui K, et al. Th17/Treg cells imbalance and GITRL profile in patients with Hashimoto's thyroiditis. Int J Mol Sci. (2014) 15:21674-86. doi: 10.3390/ijms151221674

29. Carrozzo M, Uboldi de Capei M, Dametto E, Fasano ME, Arduino P, Broccoletti R, et al. Tumor necrosis factor-alpha and interferon-gamma polymorphisms contribute to susceptibility to oral lichen planus. J Invest Dermatol. (2004) 122:87-94. doi: 10.1046/j.0022-202X.2003.22108.x

30. Brito JP, Morris JC, Montori VM.. Thyroid cancer: zealous imaging has increased detection and treatment of low risk tumours. BMJ. (2013) 347:f4706. doi: 10.1136/bmj.f4706

31. Bray F, Ferlay J, Soerjomataram I, Siegel RL, Torre LA, Jemal A. Global cancer statistics 2018: GLOBOCAN estimates of incidence and mortality worldwide for 36 cancers in 185 countries. CA Cancer J Clin. (2018) 68:394424. doi: $10.3322 /$ caac. 21492

32. Maniakas A, Davies L, Zafereo ME. Thyroid disease around the world Otolaryngol Clin North Am. (2018) 51:631-42. doi: 10.1016/j.otc.2018.01.014

Conflict of Interest: The authors declare that the research was conducted in the absence of any commercial or financial relationships that could be construed as a potential conflict of interest.

Copyright (c) 2020 Tang, Shi, Jiang, Zhou and Shen. This is an open-access article distributed under the terms of the Creative Commons Attribution License (CC BY). The use, distribution or reproduction in other forums is permitted, provided the original author(s) and the copyright owner(s) are credited and that the original publication in this journal is cited, in accordance with accepted academic practice. No use, distribution or reproduction is permitted which does not comply with these terms. 